\title{
Method of Locating End Points of Insulator Strings Based on Minimum Enclosing Rectangle
}

\author{
Jiawei Hu \\ School of North China Electric Power University Baoding, Baoding 071000, China \\ 798087749@qq.com
}

Keywords: Principal axis method; Minimum Enclosing Rectangle; Edge detection; Shape features

\begin{abstract}
In the algorithm of extracting end points of insulator strings by image technology, we propose a method to find the Minimum Enclosing Rectangle (MER) of the target by using the principal axis method based on the combination of the vertex chain code and the discrete Green theorem. Then the binary image of the extracted target is detected by edge detection and threshold segmentation, the boundary image of the insulator string is drawn. Finally, the endpoints coordinates of the insulator string are extracted based on the shape feature of insulator string. The algorithm is implemented by MATLAB software, and the results show that the method is feasible.
\end{abstract}

\section{Introduction}

In recent years, with the development of social demand and technology, the transmission lines are developing toward UHV, high-power and long-distance, along with the natural destruction and aging problem, seriously affecting the safe operation and stability of the power system. As a large amount of components in the power grid, the detection of the state of insulators on the normal operation of the power system is very necessary. Insulator tilt angle is an important monitoring quantity, its accuracy directly affects the calculation of equivalent ice thickness.

The traditional method of detecting the state of the insulator needs to be examined manually one by one, so the information acquisition equipment is relatively complex and susceptible to environmental electromagnetic interference. With the rapid development of computer vision technology and the improvement of digital camera resolution, It is an inevitable trend for the future development of the grid inspection method which combines digital camera, data acquisition and image processing technology. The non-contact measurement method ${ }^{[1]}$ can greatly improve the insulator string spatial tilt angle acquisition speed and accuracy, and extracting the insulator string endpoints from the processed image ${ }^{[2]}$ is an important part to the realization of this technology .

In this paper, a method of locating the insulator string end points based on the MER is proposed. The method can complete the fast extraction of the MER based on the combination of the vertex chain code and the discrete green ${ }^{[3]}$,and obtain geometrical features such as perimeter, area and centroid, which is convenient for the following endpoints extraction.

The flow chart is shown in Fig.1:

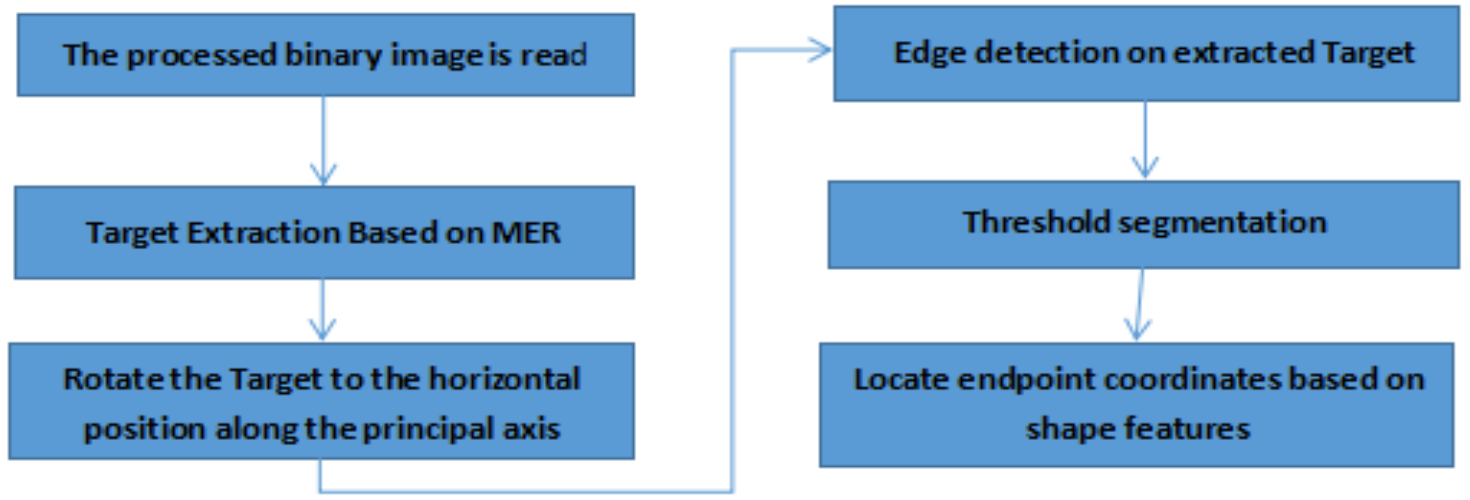

Figure.1 


\section{The algorithm is proposed}

\section{Image chain code extraction and convex set algorithm.}

The specific representation of the vertex chain code is given in References ${ }^{[4]}$.Suppose that the image size is Height $\times$ Width, the vertex matrix size is $($ Height +2$) \times($ Width +2$)$, the vertex matrix is Code;The $i-s t$ vertex walking direction is $D_{i} \in\{0,1,2,3\}$, the vertex code value is $\mathrm{C}_{\mathrm{i}} \in\{0,1,2,3\}$, the convex chain stores the bump coordinates value.

Assume that the current vertex $\mathrm{P}_{\mathrm{i}}\left(x_{\mathrm{i}}, y_{i}\right)$, The direction of travel in $\mathrm{P}_{\mathrm{i}}$ is $\mathrm{D}_{\mathrm{i}}$. The next vertex $\mathrm{P}_{\mathrm{i}+1}$ is encoded as $\mathrm{C}_{\mathrm{i}+1}$. The change rule of traveling direction is:

$$
\left\{\begin{array}{c}
\mathrm{D}_{\mathrm{i}+1}=\mathrm{D}_{\mathrm{i}}, \text { if } C_{i+1}=2 \\
\mathrm{D}_{\mathrm{i}+1}=\left(\mathrm{D}_{\mathrm{i}}+1\right) \% 4, \text { if } C_{i+1}=1 \\
\mathrm{D}_{\mathrm{i}+1}=\left(\mathrm{D}_{\mathrm{i}}+3\right) \% 4, \text { if } C_{i+1}=3
\end{array}\right.
$$

Coordinate migration rules are:

$$
\left\{\begin{array}{l}
x_{\mathrm{i}+1}=x_{\mathrm{i}+1}, y_{i+1}=y_{i}, \text { if } D_{i}=0 \\
x_{\mathrm{i}+1}=x_{\mathrm{i}}, y_{i+1}=y_{i-1}, \text { if } D_{i}=1 \\
x_{\mathrm{i}+1}=x_{\mathrm{i}-1}, y_{i+1}=y_{i}, \text { if } D_{i}=2 \\
x_{\mathrm{i}+1}=x_{\mathrm{i}}, y_{i+1}=y_{i+1}, \text { if } D_{i}=3
\end{array}\right.
$$

\section{The Combination of Discrete Green and Vertex Chain Code.}

In this paper, the vertex chain code of the image is obtained while the boundary coordinate chain and the running direction chain of the image are acquired too.By transforming the target surface integral to the target curve integral, the computational complexity of the area, centroid, and principal axis of the image is reduced ${ }^{[5]}$.

The coordinate migration rules of the direction chain :

$\mathrm{D}_{\mathrm{i}}=0: \mathrm{x}=x_{i}+t, y=y_{i}, d x=d t, d y=0$

$\mathrm{D}_{\mathrm{i}}=1: \mathrm{x}=\chi_{i}, y=y_{i}-t, d x=0, d y=-d t$

$\mathrm{D}_{\mathrm{i}}=2: \mathrm{x}=x_{i}-t, y=y_{i}, d x=-d t, d y=0$

$\mathrm{D}_{\mathrm{i}}=3: \mathrm{x}=x_{i}, y=y_{i}+t, d x=0, d y=d t$

According to Discrete Green, in the vertical direction(that is Direction $=1,3$ ) there are:

$\mathrm{P}=0, f_{1}(x, y)=\int^{x} f(x, y) d x, Q_{1}=\int_{0}^{1} f_{1}(x, y-t) d t$

$Q_{3}=-\int_{0}^{1} f_{1}(x, y+t) d t$

$\iint_{D} f(x, y) d x d y=\oint_{L}\left(Q_{1}+Q_{3}\right) d y$

In the horizontal direction(that is Direction $=0,2$ ) there are:

$\mathrm{Q}=0, f_{2}(x, y)=\int^{y} f(x, y) d y$

$P_{0}=-\int_{0}^{1} f_{2}(x+t, y) d t, P_{2}=\int_{0}^{1} f_{2}(x-t, y) d t$

$\iint_{D} f(x, y) d x d y=\oint_{L}\left(P_{0}+P_{2}\right) d y$

The principal axis of the target image is: 


$$
\tan 2 \times \alpha=\frac{2 \times \boldsymbol{u}_{11}}{\boldsymbol{u}_{20}-\boldsymbol{u}_{02}}
$$

Where $\mathrm{u}_{11}=M_{11}-y \times M_{10}, u_{20}=M_{20}-x \times M_{10}, u_{02}=M_{02}-y \times M_{01}, \alpha$ is the direction angle between the principal axis and the $\mathrm{X}$ axis.

\section{The Calculation of the Minimum Enclosing Rectangle.}

\section{1) Principal axis method}

The centroid is the coordinate origin, the principal axis is the $\mathrm{X}$ 'axis.Rotate the $\mathrm{X}$ axis, and the rotation size is the direction angle $\alpha$, and The transformation relations of the new and old coordinate systems are:

$$
\begin{aligned}
& x^{\prime}=p+\cos (\alpha) \times x+\sin (\alpha) \times y \\
& y^{\prime}=q-\sin (\alpha) \times x+\cos (\alpha) \times y
\end{aligned}
$$

Flow chart of Principal axis method is shown in Fig.2.

In the vertex chain code,the number of points in the vertical direction(Vertical DirecNum) is N1; the horizontal direction(Horizontal DirecNum) is N2.

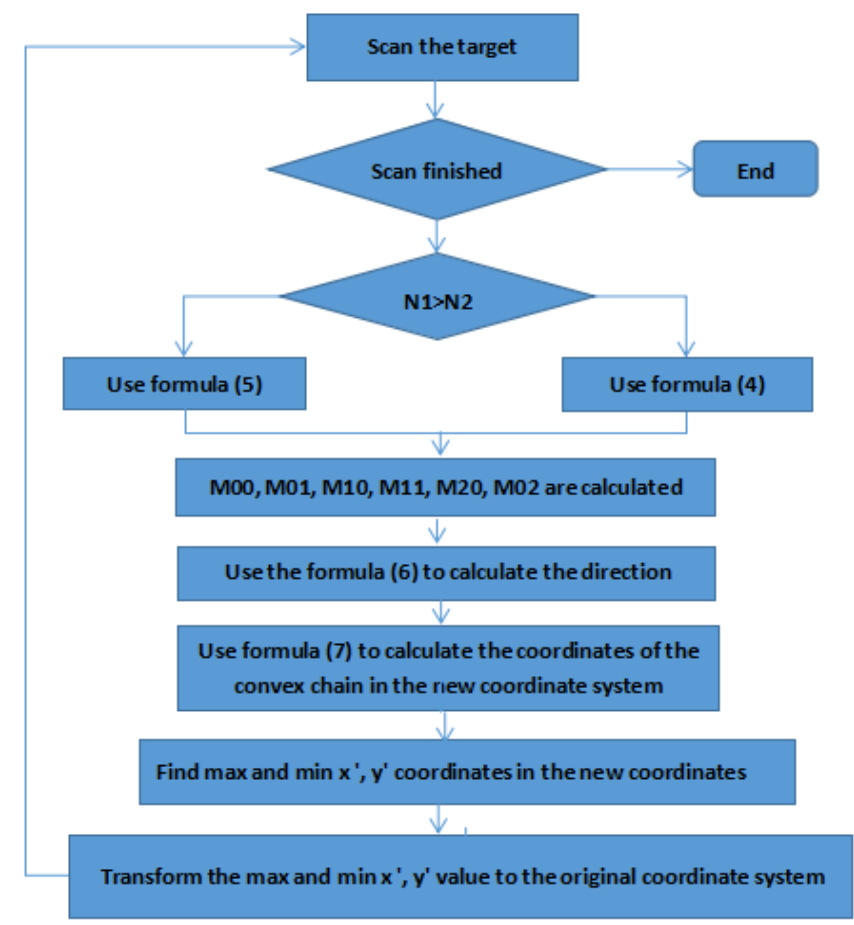

Figure.2

\section{Insulator string endpoints extraction.}

Firstly, the image is rotated by an angle $\alpha$ along the principal axis direction of the MER. Then the target is cut out for edge detection and threshold segmentation, traversal and draw the outer contour of the insulator string. Finally, based on the shape feature, the pixel coordinates of the end point of the insulator string in the horizontal state are obtained. According to the rotation angle of the image $\alpha$, through the two-dimensional coordinate transformation, we can get the two-dimensional pixel coordinates of the insulator string endpoints in the original image!

Here we select the insulator string at the beginning and end of the umbrella skirt center line and the intersection of the axis as the endpoints of the insulator string.

\section{Example verification and discussion}

Based on the above process, we achieve the above algorithm by MATLAB R2014a software. 


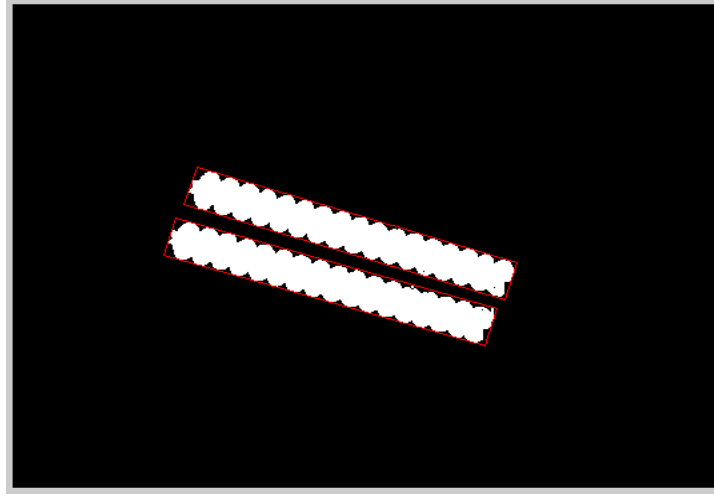

Figure.3

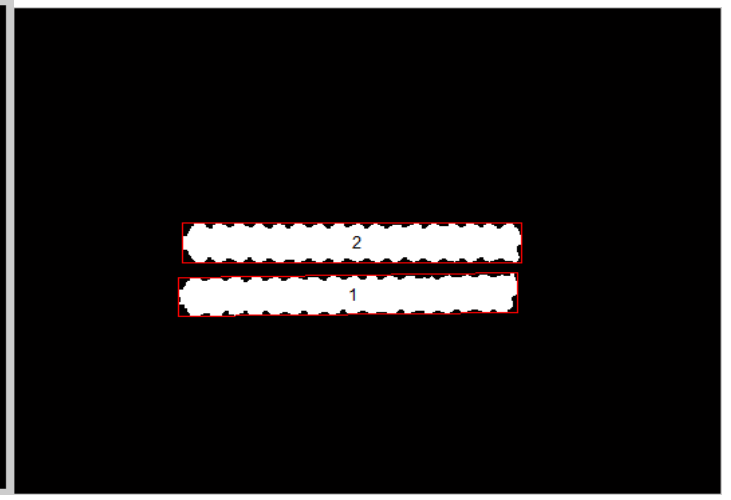

Figure.4

Firstly, read the binary image containing the target which is preprocessed, and the target is extracted with the minimum enclosing rectangle and labeled as Fig.3;Secondly,the target will be rotated to a horizontal position along the direction of its principal axis , as shown in Fig.4;Thirdly the extracted target which is cut out alone, is detected by edge detection and threshold segmentation we can clearly see the target boundary, shown in Fig.5;Finally, we can get the pixel coordinates of the endpoints of the insulator string accurately according to the geometric characteristics of the target. Marked out in the original figure, as shown in Fig. 6.

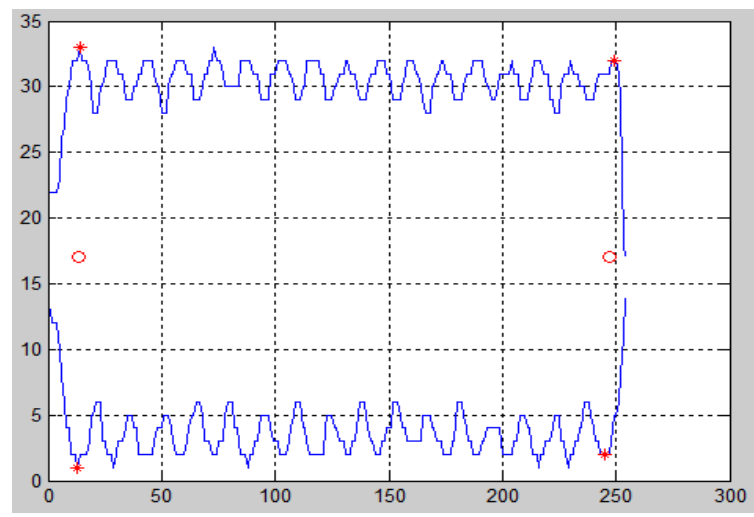

Figure.5

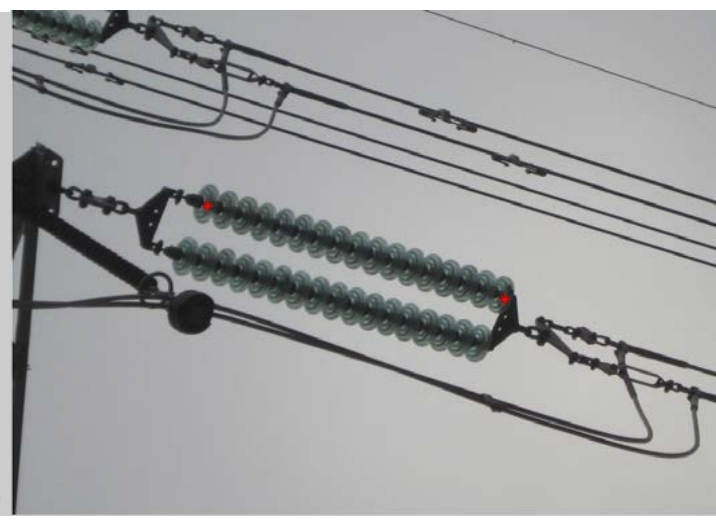

Figure.6

\section{Conclusions}

Based on the principal axis method based on chain code and Discrete Green, we can track the boundary of any target image and obtain the moment feature information of the target image, so as to realize the real time geometric feature extraction of large scale image. And the geometric features such as perimeter, area and centroid obtained in this process, are used to further perform the mathematical processing such as edge detection and threshold segmentation for the extracted target, Finally, the pixel coordinates of the end points are obtained through the shape features of insulator strings. Experiments show that this method can quickly and accurately locate the pixel coordinates of insulator string endpoints, which has certain universality and engineering application value.

\section{References}

[1] CAO Ming,ZHAO Jiankun;Research on Image Recognition Method for Angle of Insulator, YUNNAN ELECTIC POWER,Vol.44 No.2 Feb.2016

[2] YAO Chunyu,JIN Liyun,YAN Shujia.Recognition of Insulator String in Power Grid Patrol Images.Journal of System Simulation,Vol. 24 No. 9 Sep, 2012

[3] Brlek S, Labelle G, Lacasse A. The Discrete Green Theorem and Some Applications in Discrete Geometry[J]. Theoretical Computer Science, 2005, 346(2): 200-225. 
[4] WU Xiaoguang,WANG Diqiong,SHENG Hui.An Algorithm and Implementaion for Obtaining Minimum Exterior Rectangle of Image Region.Computer Engineering,Vol.30 NO.12 June 2004

[5] LU Rong,FAN Yong,CHEN Niannian.Fast Algorithm for Extracting Minimum Enclosing Rectangle of Target Image.Computer Engineering,Vol.36 No.21 November 2010 